\title{
Successful Treatment of Post-Traumatic Elbow Scar Contracture Using Combined Approach of Surgical Release \& Early Fractional Laser Resurfacing
}

\author{
Yean Su Choi \\ Galina Khan \\ Seung Min Nam \\ Eun Soo Park
}

Department of Plastic \& Reconstructive Surgery, Soonchunhyang University Bucheon Hospital, Bucheon, Korea
Received June 18, 2018

Accepted June 21, 2018

\author{
Correspondence \\ Eun Soo Park \\ Department of Plastic and Reconstructive \\ Surgery, Soonchunhyang University Bucheon \\ Hospital, 170, Jomaru-ro, Wonmi-gu, Bucheon \\ 14584, Korea \\ Tel: +82-32-621-5319 \\ Fax: +82-32-621-5662 \\ E-mail: peunsoodsschmc.ac.kr \\ (C) Korean Society for Laser Medicine and Surgery \\ (c) This is an open access article distributed under the \\ terms of the Creative Commons Attribution Non- \\ Commercial License (http://creativecommons.org/ \\ licenses/by-nc/4.0) which permits unrestricted non- \\ commercial use, distribution, and reproduction in any \\ medium, provided the original work is properly cited.
}

Scarring is mostly fibrous formation that arises from the regeneration of tissue after a traumatic injury or inflammation. One of the most common and most important morbidities of scar is contracture, particularly when located in the nearby joints. Many surgical techniques, including tissue expansion, skin grafting, local and distant flaps, and Z- or W-plasty can be applied to scar contracture. Currently, non-surgical methods play an important role in scar treatment methods with ablative fractional resurfacing being one of the most effective and promising methods for correcting various types of scar. This paper reports the combined approach of surgical and non-surgical methods of scar treatment to release scar contracture successfully. A nine-year-old male patient with a $30 \mathrm{~cm}$-sized posttraumatic scar on his right arm was treated with an ablative fractional laser device after Z-plasty. The patient received a total of four sessions of laser treatment with a one month interval between sessions. The results were evaluated before the procedure and 2 years after the last fractional laser resurfacing treatment. The mean vancouver scar scale score of the scar rated by two physicians decreased from 9.5 to 3.0. The patient also noted significant improvement in aesthetics and function and was quite satisfied with the results. No complications, such as wound disruption, post-inflammatory hyper pigmentation, or dyspigmentation were noted. The combined approach of surgical release and early fractional laser resurfacing for scar treatment can produce significant aesthetic and functional improvements of scar contracture.

\section{Key words}

Cicatrix; Hypertrophic; Lasers; Wound healing 


\section{INTRODUCTION}

Scar is mostly fibrous formation that arises from regeneration of tissue after traumatic injury or inflammation. The $40 \%$ to $70 \%$ of postoperative scars and up to $91 \%$ of burn scars are hypertrophic scars which have an excessive dermal collagen. The proportion of type of collagen in hypertrophic scar differs from that of original healthy tissue. Hypertrophic scar mainly consists of type III collagen and plenty of nodules containing myofibroblasts, large extracellular collagen filaments and abundant acidic mucopolysaccharides. ${ }^{1}$

One of the most common and most important morbidities of scar is contracture, especially located in nearby joints. Skin scarring along with tendon adhesions to the underlying musculotendinous units limits functional and motional abilities of skin. Many surgical techniques including tissue expansion, skin grafting, local and distant flaps, and Z- or W-plasty can be applied to scar contracture. Skin grafting, especially full-thickness skin graft, is simple, reliable, and safe treatment option for wide scar contracture. $^{2}$ Another practical technique for releasing scar contractures is Z-plasty. It was already proven to be safe and effective option and a few modified Z-plasty techniques were also introduced. ${ }^{3,4}$ Recently, perforatorbased interposition flap was introduced as more attractive surgical technique to release scar contracture than fullthickness skin graft. ${ }^{5}$ However, the most effective surgical technique is still controversial issue. ${ }^{6}$

Nowadays, non-surgical method plays an important role as scar treatment method and ablative fractional resurfacing (AFR) is one of the most effective and promising methods for correcting various types of scar such as acne, burn scar, postsurgical and posttraumatic scar. ${ }^{7-9}$ A laser treatment algorithm recommends applying fractional and vascular lasers to burn or traumatic scar appropriately. ${ }^{10}$ However, relatively large traumatic scars and severe scar contractures cannot be easily treated with only laser treatment. In our case, both surgical release operation and early fractional laser resurfacing are selected to treat patient's wide scar contracture. We introduce our experience of combined approach to release scar contracture successfully.

\section{PATIENT AND METHOD}

A 9-year-old male patient suffered from traumatic scar after traffic accident on his right arm. The $30 \mathrm{~cm}$-sized wide scar contracture ranged from wrist to upper arm passing through cubital fossa. The patient needed to have release of scar contracture because of severe functional disability besides aesthetic impairment. Chief complaint was the impossibility of fully straightening his right elbow. About $140^{\circ}$ was the maximal degree between patient's right forearm and upper arm when he tried to straighten right arm as much as possible.

Firstly, the scar contracture was excised sufficiently then the remnant scar tissue was released by two other Z-plasty designs in which the limbs of each design are at $60^{\circ}$ angles. All the limbs are of $2 \mathrm{~cm}$ length. The operative wound was healed successfully without any complications including infection, wound dehiscence, and necrosis. After surgical release, degree of the patient's fully straightened elbow was improved to nearly $180^{\circ}$.

Laser treatment was initiated six weeks after surgery with guardian consent. Four sessions of laser treatment using ablative carbon dioxide $\left(\mathrm{CO}_{2}\right)$ fractional laser device (LineXel ${ }^{\circledR} ;$ UTI Co. Ltd, Seoul, Korea) were performed with one month intervals. Every treatment were conducted in the outpatient clinic and were not accompanied with any sedative or systemic anesthetics. Only topical anesthetic cream leutectic mixture of $2.5 \%$ lidocaine hydrochloric acid and 2.5\% prilocaine; EMLA cream, AstraZeneca AB, Södertälje, Sweden) was applied 60-90 minutes before the procedure to reduce patient's discomfort during the treatment. The laser parameters were set as a pulse duration of 320-420 msec and a distance of 0.8-0.9 mm.

At every treatment sessions and two years after the last session, photographs of the scar were taken and range of motion of patient's right elbow was also measured. The patient and his parents were surveyed about their overall level of satisfaction using a grading scale lexcellent, good, fair, and poor). At the same time, two experienced physicians rated status of the scar using the Vancouver Scar Scale (VSS) which can evaluates four standards; pigmentation $10=$ normal, 1 = hypopigmentation, 2 = mixed pigmentation, $3=$ hyperpigmentation), pliability $(0=$ normal, 1 = supple, 2 = yielding, $3=$ firm, $4=$ banding, $5=$ contrac ture), height ( $0=$ flat, $1=<2 \mathrm{~mm}, 2=2-5 \mathrm{~mm}, 3=>5 \mathrm{~mm})$, and vascularity $\left(0=\right.$ normal, $1=$ pink, $2=$ red, $3=$ purple).$^{11}$

\section{RESULTS}

Clinically, the postoperative scar showed significant improvement after fractional $\mathrm{CO}_{2}$ laser treatment (Fig. 1). Mean VSS score for the scar was 9.5 before laser treatment and 3.0 two years after the last treatment. The patient also experienced significant improvement in scar quality and function of his elbow. Overall satisfaction of the patient and his parents was checked as 'excellent' 

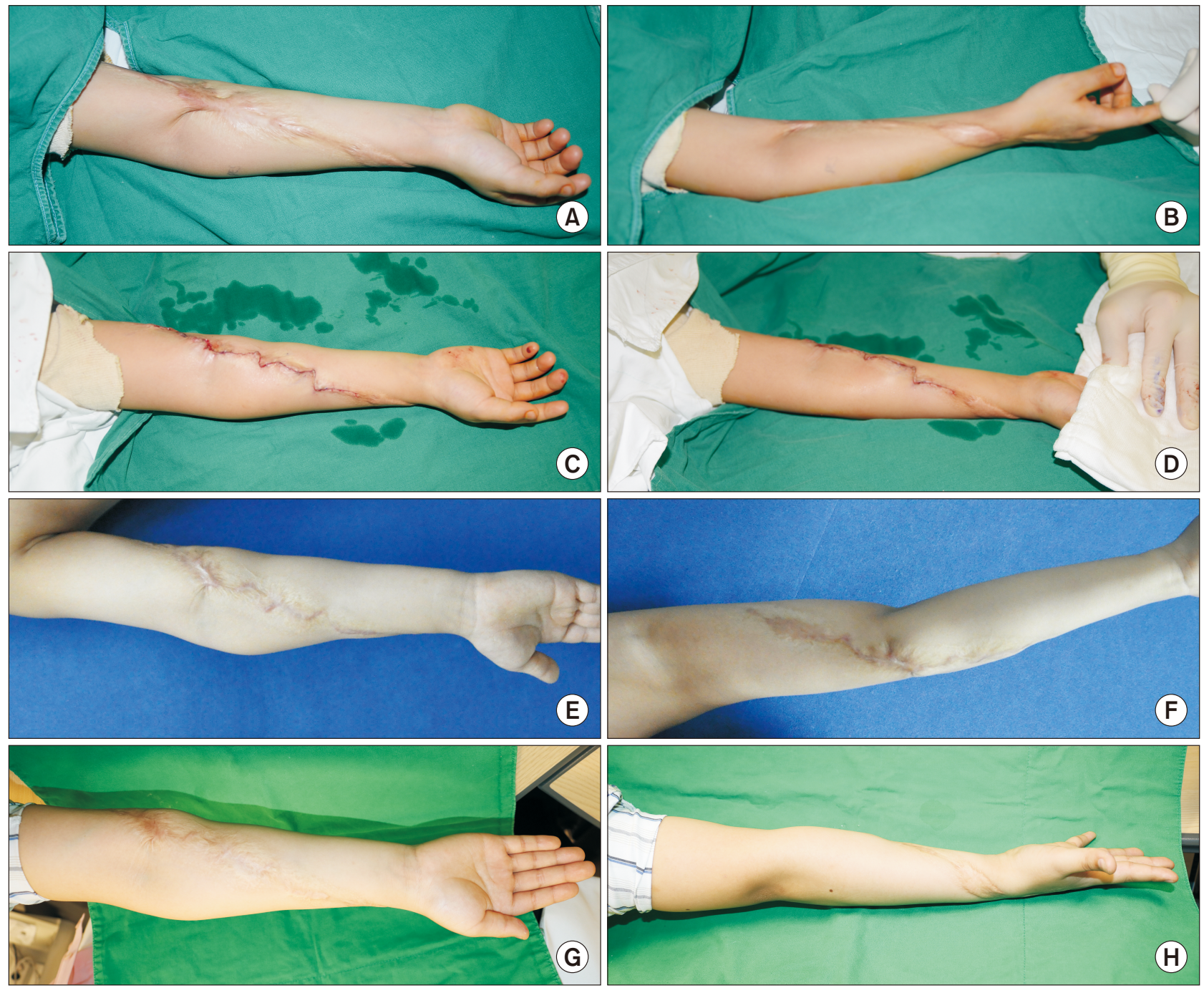

Fig. 1. Nine-year-old male patient had wide scar contracture on his right arm. (A, B) The patient couldn't straighten his right arm fully before operation. (C, D) Full extension was possible after surgical release operation. (E, F) Before fractional laser resurfacing. (G, H) 2 years after the last fractional laser resurfacing procedure.

grade. Range of motion of the right arm including extension was nearly fully recovered after surgical releasing and had not been decreased during laser sessions. From a cosmetic point, the patient and physicians noted representative improvement in both texture and pigmentation with each treatment. Two years after the final laser session, the patient was satisfied with aesthetic and functional improvement of the scar. At the end of each AFR procedure, minor complications such as erythema, pain, and heating sensation have remained for three days up to two weeks. However, any serious side effects such as postinflammatory hyperpigmentation, wound disruption, or dyspigmentation were not observed.

\section{DISCUSSION}

There are various surgical interventions and procedures that can be applied in the treatment of scars. However these manipulations frequently delayed to the scar maturation that can lead to the considerable morbidity, such as the tendon adhesions that can cause joint contractures. Contractures are one of the most common morbidities of burn and traumatic scars and it limits the range of motion of nearby joints. If there is adequate normal tissue near scar contracture and the additional bulk of the flap does not contraindicate its use, a flap is often preferable to a skin grafting for releasing scar contracture. One of the simplest and widely used methods is the random flaps, represented by Z-plastyf. ${ }^{10,12}$ Zhang et al. ${ }^{3}$ 
and Yotsuyanagi et al. ${ }^{4}$ each introduced their modified techniques of Z-plasty for releasing wide-scar contracture as useful and practical methods.

Besides surgical techniques, different types of lasers and light sources can be used in scar treatment now. Donelan et al. ${ }^{13}$ used a pulsed dye laser in combination with Z-plasty in patients with burn scars. All patients observed decreased erythema and scar thickness after laser treatments. However it was reported that ablative fractional lasers are more effective than non-ablative fractional lasers for treating hypertrophic scars. ${ }^{10}$ Fractional $\mathrm{CO}_{2}$ laser makes thousands of microscopic holes in the superficial layer of the skin. The superficial layer of a few micron thickness completely evaporates, whereas the deep layer, where the collagen fibers are located in, intensively warm up. Depth of thermal injury made by ablative fractional laser is approximately $4 \mathrm{~mm}$ which is bigger than $1.8 \mathrm{~mm}$ of non-ablative fractional laser. Injury through deeper tissue makes the ablative fractional laser as more effective for thicker scar tissues resolving limitation of motion. Thermal action of the laser activates the formation of collagen fibers, which contributes to the remodeling of the skin structure. Histologically, scars treated with fractional laser have significantly decreased type I collagen and increased type III collagen which is more similar to normal skin than untreated lesions. ${ }^{14,15}$ And what's more, vascular density in the superficial dermis is also improved. ${ }^{16}$ There are several reports about using ablative fractional laser in treatment of postsurgical and burns scars. Krakowski et a.. ${ }^{17}$ introduced his two successful cases of treating pediatric burn scar contractures with ablative fractional laser therapy. Both patients showed rapid improvement of the scar contractures especially in functional aspect. Cho et al. ${ }^{18}$ also reported that the fractional $\mathrm{CO}_{2}$ laser is an effective treatment option for facial postoperative scars without any serious adverse effects.

Although previous studies proved the usefulness and effectiveness of fractional $\mathrm{CO}_{2}$ laser, most of laser therapies were used to mature scars. In recent years, the early intervention of scars using fractional $\mathrm{CO}_{2}$ laser has received considerable attention. Early fractional resurfacing within weeks or months from injury may be helpful in preventing scar contracture formation resulting in advantages of patient rehabilitation. Ideal timing to start fractional laser treatment is not well-established yet. Traditional laser protocols avoided treating freshly healing wounds concerning unpredictable complications which can damage wound healing. Against the expectation, clinical experience reported that wounds with focal erosion or ulceration were rapidly healed after ablative fractional laser treatment. ${ }^{19}$ Based on advantages of the early intervention, several literatures recommend early laser treatment after the injury. Shumaker et al. ${ }^{20}$ initiated an ablative fractional laser treatment on patients within 2-5 months of their burns or final surgeries. All patients reported improvement of mobility and range of motion, appearance, texture and color of scars. Especially in one case, patient succeeded in regaining the complete range of motion. Hong et al. ${ }^{21}$ showed that initiating early postoperative or posttraumatic fractional $\mathrm{CO}_{2}$ laser treatment at four weeks after surgery is safe and effective. Total five treatment sessions with a month interval improved the scar quality satisfying all of the patients. In addition, Lee et al. ${ }^{22}$ performed a prospective, split-scar, evaluatorblinded study on sixteen postoperative scars. Laser procedure using $\mathrm{CO}_{2}$ fractional laser was applied twice with two-week intervals at three weeks after surgery. Three months after the last treatment, there was greater decrease of VSS score in the treated half of the scars. Author concluded $\mathrm{CO}_{2}$ fractional laser is an effective treatment modality for surgical scars in the early postoperative period.

In our case, the patient with wide scar contracture on his right arm was treated with combined approach of surgical release and early fractional laser resurfacing to induce excellent clinical outcome. We focused on functional disability caused by the scar contracture. Because removing the wide scar contractures by treating with only fractional laser was difficult, excision of the scar tissue and Z-plasty was operated first. Operation under the two Z-plasty designs released the scar contracture successfully and enabled the patient to straighten his arm completely. When he tried full extension of his right elbow as much as possible, the angle between right upper arm and forearm was increased from $140^{\circ}$ to $180^{\circ}$ after surgical release. To prevent range of motion being limited by newly produced surgical scar, ablative laser resurfacing with fractional $\mathrm{CO}_{2}$ laser was performed during early stages of scar remodeling. Total four sessions of laser treatment were initiated six weeks after surgery with one month interval between each treatment. We didn't apply long arm splint after operation to avoid restricting mobility of the elbow. Two years after the last procedure, both the patient and the surgeon satisfied with the outcome. Mean VSS score of the scar rated by two physicians were decreased from 9.5 to 3.0. The patient and his parents also rated the outcome as 'excellent' grade. One of the most meaningful points of this study is that recovered function of the patient's arm was maintained completely during total fractional laser resurfacing procedures. Although we 
introduced just one case of combined approach treating wide scar contracture, aesthetic and functional outcome was noticeable. Further experience applying newly combined approach of scar management can be helpful to establish more effective and more preferable methods for treating severe scar contractures.

\section{CONCLUSION}

Aesthetic impairment from scar contracture can significantly impact a patient, even in simple daily lives. Recently, combined approach including surgery, physical therapy, and AFR can be utilized to treat these patients. AFR is now considered the ideal choice for improving cosmetics in traumatic scarring. We were able to show significant aesthetic and functional improvement with a combined approach of surgical release and early fractional laser resurfacing. Further research may support that early intervention with AFR plays an important role in preventing development of scar contracture.

\section{DECLARATION OF INTEREST}

The authors declare that they have no conflicts of interest to disclose.

\section{ACKNOWLEDGEMENTS}

This work was supported by the Soonchunhyang University Research Fund.

\section{REFERENCES}

1. Gauglitz GG, Korting HC, Pavicic T, Ruzicka T, Jeschke MG. Hypertrophic scarring and keloids: pathomechanisms and current and emerging treatment strategies. Mol Med 2011;17:11325.

2. Iwuagwu FC, Wilson D, Bailie F. The use of skin grafts in postburn contracture release: a 10-year review. Plast Reconstr Surg 1999;103:1198-204.

3. Zhang L, Jin R, Shi YM, Sun BS, Pu ZM, Zhang YG. Reversed $Z$-plasty and its variations to release wide-scar contraction. Burns 2014:40:1185-8.

4. Yotsuyanagi T, Yamashita K, Gonda A, Kato S, Sugai A, Yamada $T$, et al. Double combined Z-plasty for wide-scar contracture release. J Plast Reconstr Aesthet Surg 2013;66:629-33.

5. Stekelenburg CM, Jaspers ME, Jongen SJ, Baas DC, Gardien $\mathrm{KL}$, Hiddingh J, et al. Perforator-based interposition flaps perform better than full-thickness grafts for the release of burn scar contractures: a multicenter randomized controlled trial.
Plast Reconstr Surg 2017;139:501e-9e.

6. Stekelenburg CM, Marck RE, Tuinebreijer WE, de Vet HC, Ogawa R, van Zuijlen PP. A systematic review on burn scar contracture treatment: searching for evidence. J Burn Care Res 2015;36:e153-61.

7. Waibel J, Beer K. Ablative fractional laser resurfacing for the treatment of a third-degree burn. J Drugs Dermatol 2009;8:294-7.

8. Weiss ET, Chapas A, Brightman L, Hunzeker C, Hale EK, Karen JK, et al. Successful treatment of atrophic postoperative and traumatic scarring with carbon dioxide ablative fractional resurfacing: quantitative volumetric scar improvement. Arch Dermatol 2010;146:133-40.

9. Cervelli V, Gentile P, Spallone D, Nicoli F, Verardi S, Petrocelli $\mathrm{M}$, et al. Ultrapulsed fractional CO2 laser for the treatment of post-traumatic and pathological scars. J Drugs Dermatol 2010;9:1328-31.

10. Anderson RR, Donelan MB, Hivnor C, Greeson E, Ross EV, Shumaker PR, et al. Laser treatment of traumatic scars with an emphasis on ablative fractional laser resurfacing: consensus report. JAMA Dermatol 2014;150:187-93.

11. Baryza MJ, Baryza GA. The Vancouver Scar Scale: an administration tool and its interrater reliability. J Burn Care Rehabil 1995; 16:535-8.

12. Kroonen L, Shumaker PR, Kwan JM, Uebelhoer N, Hofmeister E. Treatment of split-thickness skin graft-related forearm scar contractures with a carbon dioxide laser protocol: 3 case reports. J Hand Surg Am 2013;38:2164-8.

13. Donelan MB, Parrett BM, Sheridan RL. Pulsed dye laser therapy and z-plasty for facial burn scars: the alternative to excision. Ann Plast Surg 2008;60:480-6.

14. Hantash BM, Bedi VP, Kapadia B, Rahman Z, Jiang K, Tanner H, et al. In vivo histological evaluation of a novel ablative fractional resurfacing device. Lasers Surg Med 2007;39:96-107.

15. Ozog DM, Liu A, Chaffins ML, Ormsby AH, Fincher EF, Chipps LK, et al. Evaluation of clinical results, histological architecture, and collagen expression following treatment of mature burn scars with a fractional carbon dioxide laser. JAMA Dermatol 2013;149:50-7.

16. Connolly KL, Chaffins M, Ozog D. Vascular patterns in mature hypertrophic burn scars treated with fractional C02 laser. Lasers Surg Med 2014;46:597-600.

17. Krakowski AC, Goldenberg A, Eichenfield LF, Murray JP, Shumaker PR. Ablative fractional laser resurfacing helps treat restrictive pediatric scar contractures. Pediatrics 2014;134:e17005.

18. Cho SE, Nam SM, Kim YB, Park ES. Local flap surgical scar management caused by skin cancer using fractional $\mathrm{CO} 2$ laser treatment. Arch Aesthetic Plast Surg 2015;21:18-22.

19. Shumaker PR, Kwan JM, Badiavas EV, Waibel J, Davis S, 
Uebelhoer NS. Rapid healing of scar-associated chronic wounds after ablative fractional resurfacing. Arch Dermatol 2012;148:1289-93.

20. Shumaker PR, Kwan JM, Landers JT, Uebelhoer NS. Functional improvements in traumatic scars and scar contractures using an ablative fractional laser protocol. J Trauma Acute Care Surg 2012;73:S116-21.
21. Hong SC, Park ES, Kim YB, Nam SM. Effects of minimizing scar formation by early fractional $\mathrm{CO} 2$ laser resurfacing. Arch Aesthetic Plast Surg 2014;20:109-13.

22. Lee SH, Zheng Z, Roh MR. Early postoperative treatment of surgical scars using a fractional carbon dioxide laser: a splitscar, evaluator-blinded study. Dermatol Surg 2013;39:1190-6. 